\title{
Optimal Packings of Superballs
}

\author{
Y. Jiao ${ }^{1}$, F. H. Stillinger ${ }^{2}$ and S. Torquato ${ }^{2,3,4,5}$ \\ ${ }^{1}$ Department of Mechanical and Aerospace Engineering, \\ Princeton University, Princeton New Jersey 08544, USA \\ ${ }^{2}$ Department of Chemistry, Princeton University, \\ Princeton New Jersey 08544, USA \\ ${ }^{3}$ Program in Applied and Computational Mathematics, \\ Princeton University, Princeton New Jersey 08544, USA \\ ${ }^{4}$ School of Natural Sciences, Institute for Advanced Study, Princeton NJ 08540 and \\ ${ }^{5}$ Princeton Center for Theoretical Science, \\ Princeton University, Princeton New Jersey 08544, USA
}

(Dated: October 24, 2018) 


\begin{abstract}
Dense hard-particle packings are intimately related to the structure of low-temperature phases of matter and are useful models of heterogeneous materials and granular media. Most studies of the densest packings in three dimensions have considered spherical shapes, and it is only more recently that nonspherical shapes (e.g., ellipsoids) have been investigated. Superballs (whose shapes are defined by $\left.\left|x_{1}\right|^{2 p}+\left|x_{2}\right|^{2 p}+\left|x_{3}\right|^{2 p} \leq 1\right)$ provide a versatile family of convex particles $(p \geq 0.5)$ with both cubic- and octahedral-like shapes as well as concave particles $(0<p<0.5)$ with octahedrallike shapes. In this paper, we provide analytical constructions for the densest known superball packings for all convex and concave cases. The candidate maximally dense packings are certain families of Bravais lattice packings (in which each particle has 12 contacting neighbors) possessing the global symmetries that are consistent with certain rotational symmetries of a superball. We also provide strong evidence that our packings for convex superballs $(p \geq 0.5)$ are most likely the optimal ones. The maximal packing density as a function of $p$ is nonanalytic at the sphere-point $(p=1)$ and increases dramatically as $p$ moves away from unity. Two more nontrivial nonanalytic behaviors occur at $p_{c}^{*}=1.1509 \ldots$ and $p_{o}^{*}=\ln 3 / \ln 4=0.7924 \ldots$ for "cubic" and "octahedral" superballs, respectively, where different Bravais lattice packings possess the same densities. The packing characteristics determined by the broken rotational symmetry of superballs are similar to but richer than their two-dimensional "superdisk" counterparts [Y. Jiao, F. H. Stillinger and S. Torquato, Phys. Rev. Lett., 100, 245504 (2008)], and are distinctly different from that of ellipsoid packings. Our candidate optimal superball packings provide a starting point to quantify the equilibrium phase behavior of superball systems, which should deepen our understanding of the statistical thermodynamics of nonspherical-particle systems.
\end{abstract}

PACS numbers: $61.50 . \mathrm{Ah}, 05.20 . \mathrm{Jj}$ 


\section{INTRODUCTION}

Packing problems, such as how densely given solid objects can fill $d$-dimensional Euclidean space $\Re^{d}$, have been a source of fascination to mathematicians and scientists for centuries, and continue to intrigue them today. Dense packings of hard particles have served as useful models to understand the structures of low-temperature phases of matter, such as liquids, glasses and crystals [1, 2, 4], heterogeneous materials [3, 4, 5], granular media [6, 7], and even protein folding [8]. Packing problems arise in many different branches of pure mathematics [9, 10] and in information theory [11].

In general, a collection of given solid objects (particles) in $d$-dimensional Euclidean space $\mathbb{R}^{d}$ is called a packing if no two of the objects have an interior point in common. The packing density $\phi$ is defined as the fraction of space $\mathbb{R}^{d}$ covered by the particles. A problem of great interest is the determination of the densest arrangement(s) of such particles and the associated maximal density $\phi_{\max }$. Besides the aforementioned applications, finding maximal density packings is of importance to understanding the structure and properties of crystalline equilibrium phases of particle systems as well as their ground-state $(T=0)$ structures in low dimensions in which the interactions are characterized by steep repulsions and short-ranged attractions.

The optimal solution to this packing problem for congruent particles that do not tile space is already very challenging, even in low-dimensional Euclidean spaces $\mathbb{R}^{d}(d=2,3)$. In two dimensions, the optimal packing of circular disks is the well-known triangular-lattice packing with density $\phi_{\max }=\pi /(2 \sqrt{3})$. For ellipses, the densest packing can be obtained by an affine transformation of the triangular-lattice packing of circular disks, which possesses the same density $\phi_{\max }=\pi /(2 \sqrt{3})$. These packings can also be constructed by enclosing each particle with a hexagon with minimum area that tessellates $\mathbb{R}^{2}[12,13]$. It is only recently that the famous Kepler conjecture, which postulates that the densest packing of spheres in $\mathbb{R}^{3}$ has a density $\phi_{\max }=\pi /(3 \sqrt{2})$, as realized by the stacking variants of the face-centered cubic (FCC) lattice packing, has been proved [14]. Although dense sphere packings for $d \geq 4$ have received considerable recent attention [15, 16, 17, 18, 19], optimal solutions are not rigorously known. Packing problems in high Euclidean dimensions are intimately related to the best way of transmitting digital signals over a noisy channel [10, 11].

Understanding the organizing principles that lead to the densest packings of nonspherical 
particles that do not tile space is of great practical and fundamental interest. Clearly, the effect of asphericity is an important feature to include on the way to characterizing more fully real dense granular media. Another important application relates to supramolecular chemistry [20] of organic compounds whose molecular constituents can possess many different types of group symmetries [21].

On the theoretical side, no results exist that rigorously prove the densest packings of other congruent non-space-tiling particles in three dimensions. For congruent ellipsoids, the densest known packings with density $\phi_{\max }=0.7707 \ldots$ are achieved by a family of crystal packings (for a wide range of aspect ratios) in which each ellipsoid has contact with 14 others [22]. Recently, Conway and Torquato found dense periodic packings of regular tetrahedra with $\phi \approx 0.72$ by filling imaginary icosahedra with the densest arrangement of 20 tetrahedra and arranging the icosahedra in their optimal Bravais lattice arrangement [23]. Chaikin et al. experimentally produced jammed disordered packings of nearly tetrahedral dice with density $\phi \approx 0.75[24]$. Chen has discovered the densest known periodic packing of tetrahedra with $\phi=0.7786 \ldots[25,26]$.

This paper is concerned with superball packings in low dimensions, although primarily in three dimensions. A $d$-dimensional superball is a centrally symmetric body in $\mathbb{R}^{d}$ occupying the region [27]

$$
\left|x_{1}\right|^{2 p}+\left|x_{2}\right|^{2 p}+\cdots+\left|x_{d}\right|^{2 p} \leq 1,
$$

where $x_{i}(i=1, \ldots, d)$ are Cartesian coordinates and $p \geq 0$ is the deformation parameter, which indicates to what extent the particle shape has deformed from that of a $d$-dimensional sphere $(p=1)$. A particle is centrally symmetric if it has a center $P$ that bisects every chord through $P$ connecting any two boundary points of the particle. We note that a particle is convex if the entire line segment connecting two points of the particle also belongs to the particle; otherwise it is concave. The terms superdisk and superball will be our designations for the two-dimensional $(d=2)$ and three-dimensional $(d=3)$ cases, respectively. In general, a superdisk possesses square symmetry, as $p$ moves away from unity, two families of superdisks with square symmetry can be obtained, with the symmetry axes rotated 45 degrees with respect to each other; when $p<0.5$, the superdisk is concave (see Fig. (1).

In three dimensions, a superball is a perfect sphere at $p=1$, but can possess two types of shape anisotropy: cubic-like shapes (three-dimensional analog of the square symmetry of the superdisk) and octahedral-like shapes, depending on the value of the deformation 


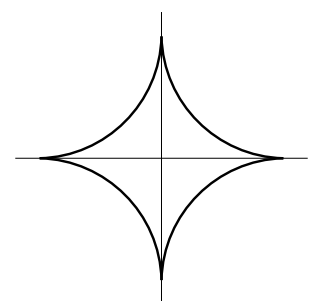

(a) $\mathrm{p}=0.45$

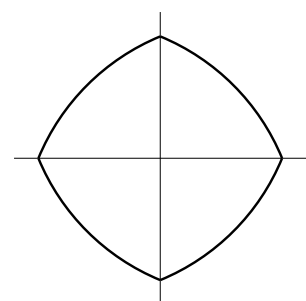

(b) $\mathrm{p}=0.75$

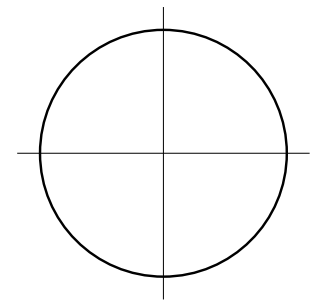

(c) $\mathrm{p}=1.0$

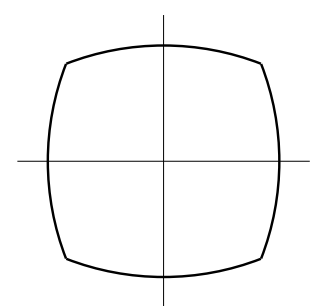

(d) $\mathrm{p}=2.0$

FIG. 1: Superdisks with different values of the deformation parameter $p$.

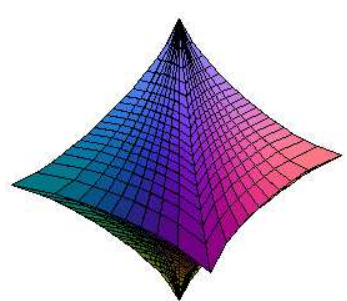

(a) $\mathrm{p}=0.45$

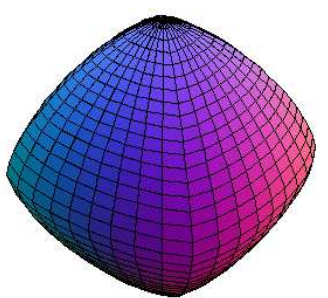

(b) $\mathrm{p}=0.75$

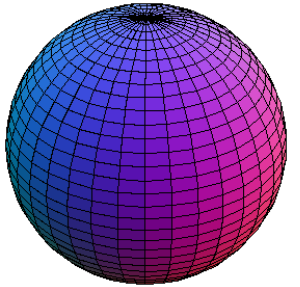

(c) $\mathrm{p}=1.0$

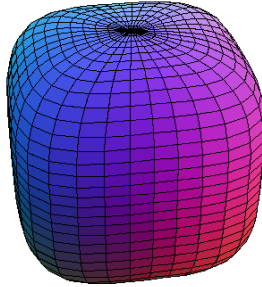

(d) $\mathrm{p}=2.0$

FIG. 2: (color online). Superballs with different values of the deformation parameter $p$.

parameter $p$ (see Fig. 2). As $p$ continuously increases from 1 to $\infty$, we have a family of convex superballs with cubic-like shapes; at the limit $p=\infty$, the superball is a perfect cube. As $p$ decreases from 1 to 0.5 , a family of convex superballs with octahedral-like shapes are obtained; at $p=0.5$, the superball becomes a regular octahedron. When $p<0.5$, the superball still possesses an octahedral-like shape but is now concave, becoming a threedimensional "cross" in the limit $p \rightarrow 0$ [28]. Note that the cube and regular octahedron (two of the five Platonic polyhedra) have the same group symmetry (i.e., they have the same 48 space group elements) because they are dual to each other [29].

Recently, we constructed the densest known packings of superdisks in $\mathbb{R}^{2}$, which provides a wide class of packings of both convex and concave particles with square symmetry [30]. In particular, the optimal packing is achieved by one of two families of Bravais lattice packings, which we called the $\Lambda_{0^{-}}$and $\Lambda_{1}$-lattice packings. (Note that in a Bravais lattice packing there is only one particle in the fundamental cell in contrast to periodic packings that have multiparticle fundamental cells.) In the following, we will usually use the term "lattice" to mean a "Bravais lattice." As the particle shape moves away from the circular disk point, the packing density increases dramatically from $\pi /(2 \sqrt{3})$ and shows a non-analytic behavior at 
the circular point. Here we stress that in light of a theorem due to Fejes Tóth [12, 13], which states that the optimal packing of any centrally symmetric convex body in $\mathbb{R}^{2}$ can be always achieved by circumscribing the body with the smallest hexagon that tiles the plane, our packings can be verified to be optimal for every specific value of $p>0.5$. This procedure is outlined in the Appendix of this paper.

In this paper, we construct the densest known packings of convex superballs, which are suggested by simulations and based on the requirements that certain group symmetries of the packings are preserved as the superballs deform from the sphere and octahedron points. Moreover, we show that as $p$ changes from unity, i.e., moves away from the sphere point, maximal packing density $\phi_{\max }$ rises steeply. The broken rotational symmetry of superballs results in a cusp in $\phi_{\max }$ at $p=1$. Thus, the initial increase of $\phi_{\max }$ is linear in $|p-1|$ and $\phi_{\max }$ is a nonanalytic function of $p$ at $p=1$. Two more nontrivial nonanalytic behaviors occur at $p_{c}^{*}=1.1509 \ldots$ and $p_{o}^{*}=\ln 3 / \ln 4=0.7924 \ldots$ for "cubic" and "octahedral" superballs, respectively,

For superballs in the cubic regime $(p>1)$, the candidate optimal packings are achieved by two families of Bravais lattice packings possessing two-fold (see Sec. III.A) and threefold rotational symmetry (see Sec. III.B), respectively, which can both be considered to be continuous deformations of the FCC lattice. For superballs in the octahedral regime $(0.5<p<1)$, there are also two families of Bravais lattices obtainable from continuous deformations of the FCC lattice keeping its four-fold rotational symmetry (see Sec. III.B), and from the densest lattice packing for regular octahedra [32, 33], keeping the translational symmetry of the projected lattice on the coordinate planes, which are apparently optimal in the vicinity of the sphere point and the octahedron point, respectively (see Secs. IV.A and IV.B). The fact that the two families of lattice packings for superballs with octahedral shapes constructed separately from the provable optimal packing of spheres and the densest known packing of octahedra (which we conjecture to be optimal [34]) meet at a single point (the same packing density with the same $p$ value) strongly suggests that our candidate packings are most likely optimal. The packing characteristics determined by the broken rotational symmetry of superballs are similar to but richer than their two-dimensional "superdisk" counterparts [30] and are distinctly different from that of ellipsoid packings [22].

For concave superballs $(p<0.5)$, the lack of simulation techniques to generate such dense packings prevent us from drawing definitive conclusions about the optimality of concave 
superball packings. Here we construct a family of dense packings of concave superballs that are close to optimal around the octahedron point $(p=0.5)$, based on the densest Bravais lattice packing and will discuss an interesting aligning effect in the limit $p \rightarrow 0$ (see Sec. IV.C).

The rest of the paper is organized as follows: In Sec. II, we briefly describe the simulation techniques used to generate dense packings of superballs, which guide our analytical packing constructions. In Sec. III and Sec. IV, we construct the densest known packings of superballs in the cubic and octahedral regimes, respectively. The structural characteristics of the packings and the affect of the broken symmetry of the superballs are discussed in detail. In Sec. V, we present concluding remarks. In the Appendix, we briefly outline the procedure to show that for every specific value of $p$ the constructed packings of two-dimensional superdisks in Ref. [30] are indeed optimal.

\section{DENSE PACKINGS OF SUPERBALLS VIA COMPUTER SIMULATIONS}

\section{A. Event-Driven Molecular Dynamics Algorithm for Superballs in the Cubic Regime}

Recently, Donev, Torquato and Stillinger developed a highly efficient event-driven molecular dynamics packing algorithm [35], which generalizes the Lubachevsky-Stillinger (LS) sphere-packing algorithm [36] to the case of other centrally symmetric convex bodies (e.g., ellipsoids and superballs). Henceforth, we refer to the algorithm as the Donev-TorquatoStillinger (DTS) algorithm for convenience. The DTS algorithm works as follows: Initially, small particles (in our case the superballs) are randomly distributed and randomly oriented in the simulation box (fundamental cell) with periodic boundary conditions and without any overlap. The particles are then given translational and rotational velocities randomly and their motion followed as they collide elastically and also expand uniformly, while the fundamental cell deforms to better accommodate the configuration. After some time, a jammed state with a diverging collision rate $\gamma$ is reached and the density reaches a local maximum value.

In order to generate the densest packing (or a packing close to the densest one) of superballs in the cubic regime $(p>1)$, a sufficiently slow growth rate is necessary, as verified 


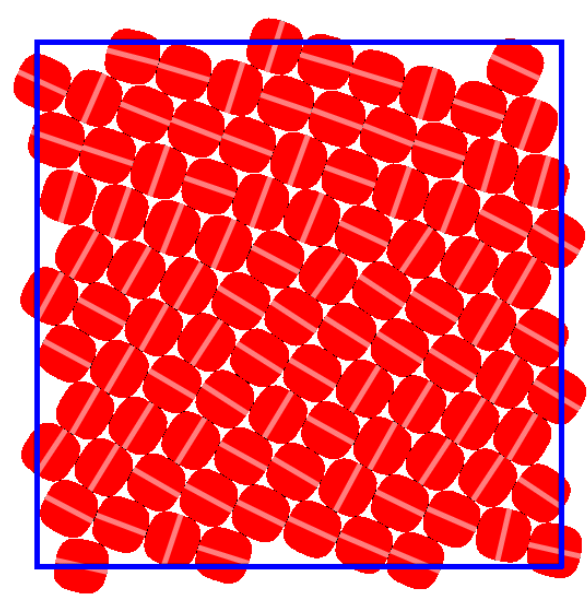

(a)

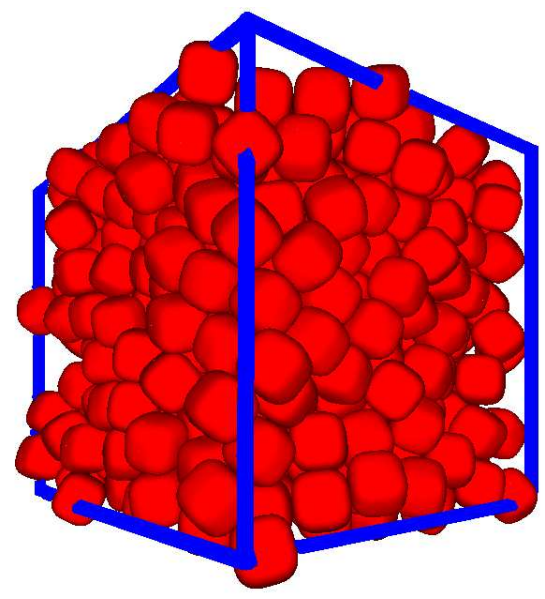

(b)

FIG. 3: (color online). Dense packings of superdisks (a) and superballs (b) with $p=1.5$ generated via the Donev-Torquato-Stillinger algorithm with the growth rate $\gamma=10^{-5}$. The packing densities are $\phi_{a}=0.9123 \ldots$ and $\phi_{b}=0.7072 \ldots$, respectively. One can see that the superdisk packing is nearly completely crystallized, while the superball packing shows no significant signs of crystallization. The white "chord" in each superdisk indicates one of its symmetry axis.

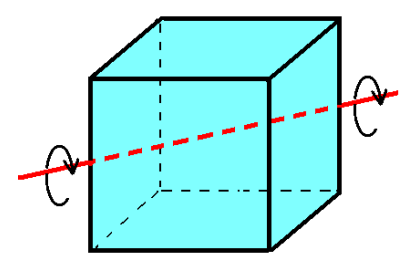

(a)

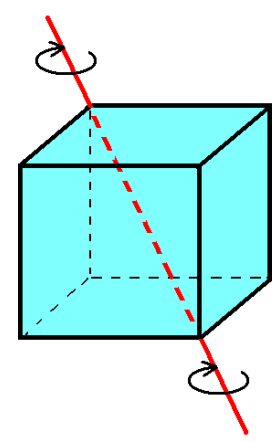

(b)

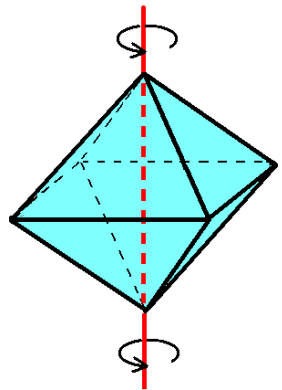

(c)

FIG. 4: (color online). Illustrations of certain rotational symmetries of the cube and regular octahedron. (a) Two-fold rotational symmetry of a cube (axis shown coincides with a face diagonal). (b) Three-fold rotational symmetry of a cube (axis shown coincides with a body diagonal). Four-fold rotational symmetry of a regular octahedron. 
by the extensive studies on spheres and circular disks, ellipsoids and ellipses as well as superdisks [22, 30, 37, 38]. In two dimensions, the densest local packing of many centrally symmetric convex particles (among which circular disks, ellipses and superdisks are of particular interest) can tessellate the space. The consistency of local and global optima results in nearly complete crystallization in large packings of these particles [e.g., see Fig. 33(a)]. By contrast, in three dimensions the geometrical frustration of superballs (i.e., the densest local packing is not consistent with the optimal global packing) makes it very difficult for the system to follow the equilibrium branch of the phase diagram (without becoming stuck in any local minima) all the way to the densest packing state, even when small growth rates are used [e.g., see Fig. [3(b)].

To resolve this difficulty, one could specify initial configurations that are not random. A good initial configuration would be unsaturated [39] lattice packings that are hypothesized to be similar to the optimal lattice packing, which can be obtained by a reasonable guess. Here we choose to arrange the superballs on the Bravais lattices that provide dense packings of spheres, such as the face-centered cubic lattice and its stacking variants as well as the body-centered cubic lattice, with a four-fold rotational symmetry axis of a superball that is parallel to a coordinate axis.

Two kinds of highly dense lattice packings of superballs emerge from the simulations [see Fig. 6(a) and (b)] starting from the unsaturated face-centered cubic packing as the initial configuration. Subsequent analytical calculations suggested by these simulation results lead us to the exact construction of two families of Bravias lattice packings, which we call the $\mathbb{C}_{0^{-}}$and $\mathbb{C}_{1}$-lattice packings (so named because they both involve particles with cubic-like shapes), whose global symmetries are consistent with the two-fold [see Fig 4(a)] and threefold rotational symmetry [see Fig. 4(b)] of a superball of the packing, respectively. The structural and packing characteristics of the $\mathbb{C}_{0^{-}}$and $\mathbb{C}_{1}$-lattice packings will be discussed in detail in Sec. III. We emphasize here that we do not exclude the possibility of the existence of denser periodic packings with a complex particle basis, although we did not find any of these packings by running the simulations with a small number of particles in the fundamental cell which facilitates occurrence of denser periodic packings if they existed [22]. 


\section{B. Stochastic Optimization Algorithm for Superballs in Octahedral Regime}

For convex superballs in the octahedral regime $(0.5<p<1)$, the prediction of the collision sequence of particles, an essential step in the DTS algorithm, is numerically unstable when the particle shape deviates too much from a sphere [see Fig. 2(b)] [40]. Here we use a novel stochastic optimization packing algorithm [41] to generate dense packings of convex superballs with octahedral-like shapes. Starting with a given initial configuration, the positions and orientations of the particles as well as the lattice vectors are considered as "design variables", on which the packing density is dependent. Various optimization techniques could be used to search the design-variable space for a point associated with a (local) maximum of packing density close to the initial value, subject to the nonoverlapping conditions. This process is repeated and the packing density is gradually increased until a global maximum is reached. For superballs, a stochastic optimization technique is employed whereby random small trial moves in the design-variable space are generated such any move is accepted if it leads to an increase of the packing density without causing any overlaps, and is rejected otherwise.

The final density of the packing generated via the optimization algorithm is sensitively dependent on the initial configuration. Thus, appropriate choices of initial configurations are crucial to the determination of the optimal packings. Based on the results of packings of superdisks and superballs in the cubic regime, it is reasonable to assume that the optimal packing lattice deforms continuously as the shape of the superball changes, while the group symmetry of the lattice consistent with the four-fold rotational symmetry of superballs is preserved. Near the sphere point, we take advantage of the cubic two-fold rotational symmetry of the FCC lattice by orienting the superballs with their four-fold rotational symmetry axis parallel to those of the lattice (see Fig. 12). Near the octahedron point, we use Minkowski's densest lattice packing for regular octahedra [32] (with the density $\left.\phi_{M}=18 / 19=0.9473 \ldots\right)$ and insert a shrunken superball into each octahedron in the packing. Note that Minkowski's lattice packing is the densest known packing of regular octahedra, which we conjecture to be optimal among all packings of regular octahedra, as

explained in [34]. The unsaturated packings so constructed are used as initial configurations for the optimization algorithm.

Two types of highly dense lattice packings emerge from the simulations [see Fig. 6(c) 


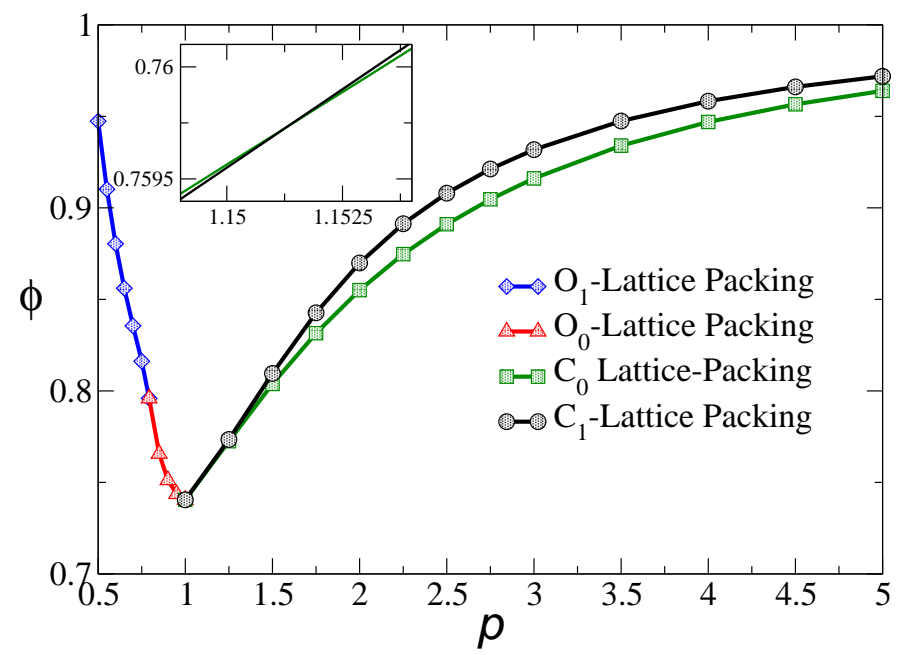

FIG. 5: (color online). Density versus deformation parameter $p$ for the packings of convex superballs. Insert: Around $p_{c}^{*}=1.1509 \ldots$, the two curves are almost locally parallel to each other.

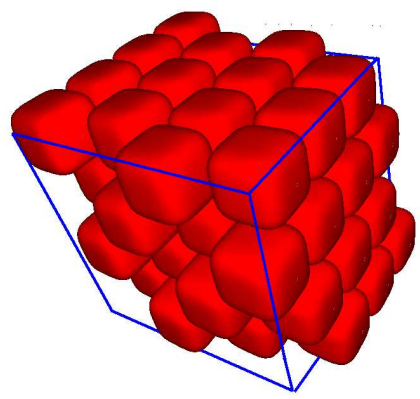

(a)

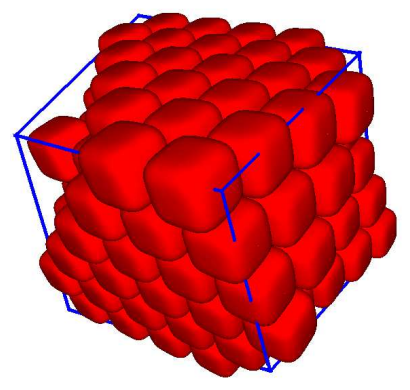

(b)

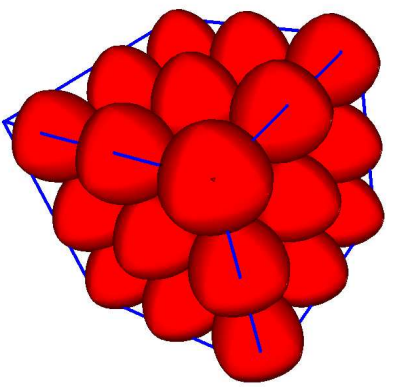

(c)

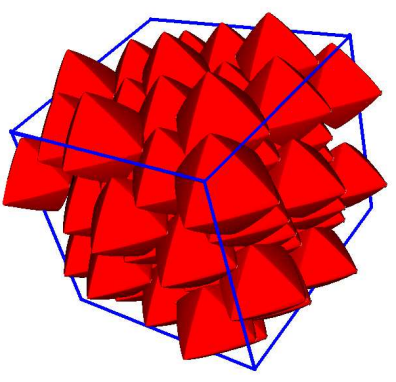

(d)

FIG. 6: (color online). Candidate optimal packings of superballs: (a) The $\mathbb{C}_{0}$-lattice packing of superballs with $p=1.8$. (b) The $\mathbb{C}_{1}$-lattice packing of superballs with $p=2.0$. (c) The $\mathbb{O}_{0}$-lattice packing of superballs with $p=0.8$. (d) The $\mathbb{O}_{1}$-lattice packing of superballs with $p=0.55$.

and $(\mathrm{d})]$, which we call the $\mathbb{O}_{0^{-}}$and $\mathbb{O}_{1}$-lattice packings, respectively (so named because the particles have octahedral-like shapes). The packings and their structural characteristics will be discussed in detail in Sec. IV. 


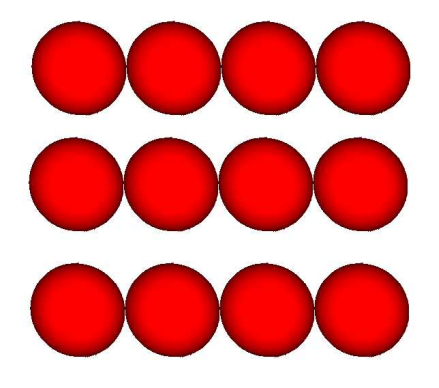

(a)

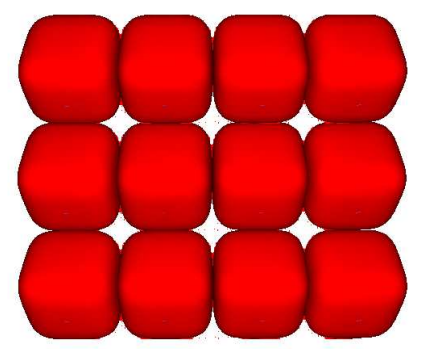

(b)

FIG. 7: (color online). (a) The stretched square-lattice layer of spheres. (b) The stretched lattice layer of superballs.

\section{OPTIMAL PACKING OF SUPERBALLS IN THE CUBIC REGIME}

\section{A. The $\mathbb{C}_{0}$-Lattice Packing}

As suggested by the simulation results, our analysis shows that the densest packings of superballs with $1<p<p_{c}^{*}$ are given by the $\mathbb{C}_{0}$ lattices, where $p_{c}^{*}=1.1509 \ldots$ with $\phi_{c}^{*}=0.7596 \ldots$ Although the $\mathbb{C}_{0}$-lattice packings are only apparently optimal for $p \in\left(1, p_{c}^{*}\right)$, they exist for all $p \geq 1$, contrary to the $\mathbb{O}_{0^{-}}$and $\mathbb{O}_{1}$-lattice packings of superballs with octahedral-like shapes, which only exist for certain range of $p$ as discussed below. The $\mathbb{C}_{0}$-lattice is obtainable from continuous deformation of the face-centered cubic lattice. In particular, the FCC lattice packing can be considered as a laminate of square-lattice planar layers [see Fig. 7(a)]. The planar square-lattice must be stretched along one of its orthogonal directions so that the superballs in the cubic regime can be arranged on the (stretched) lattice site with one of its two-fold rotational symmetry axis along the stretched direction and the other one perpendicular to the plane of the square lattice, as shown in Fig. 7(b). The magnitude of stretching is determined by the shape of the superballs, thus by the $p$ value. The stretched layers are then stacked so that the superballs in the top layers can sit exactly in the "pockets" formed by every four neighboring superballs in the bottom layer. Thus, each superball has 4 contacting neighbors in its own layers, 4 in the layer above and 4 in the layer below. The layer lamination is continued ad infinitum to construct the packing. 


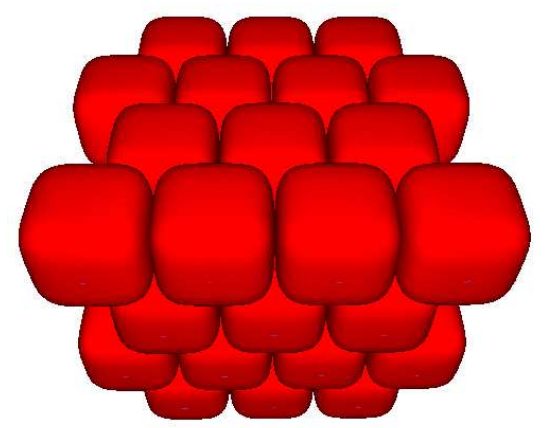

FIG. 8: (color online). The $\mathbb{C}_{0}$ lattice for superballs in the cubic regime viewed from the [110] direction. The structure remains invariant when it is rotated $180^{\circ}$ about an axis in the [110] direction, showing its two-fold rotational symmetry.

The above construction uniquely defines the $\mathbb{C}_{0}$-lattice packings which possess two-fold rotational symmetry [see Fig. 8] consistent with that of the superballs [see Fig. 4(a)]. The lattice vectors for any $p$ value are given by

$$
\mathbf{e}_{1}=2^{1-\frac{1}{2 p}} \mathbf{i}+2^{1-\frac{1}{2 p}} \mathbf{j}, \quad \mathbf{e}_{2}=2 \mathbf{k}, \quad \mathbf{e}_{3}=-2 s \mathbf{i}+2\left(s+2^{-\frac{1}{2 p}}\right) \mathbf{j}+\mathbf{k},
$$

where $\mathbf{i}, \mathbf{j}$ and $\mathbf{k}$ are the unit vectors along the coordinate axis and $s$ is the smallest positive root of the following equation:

$$
\left(s+2^{-\frac{1}{2 p}}\right)^{2 p}+s^{2 p}+2^{-2 p}-1=0 .
$$

The packing density $\phi_{\max }$ is given by

$$
\phi=\frac{V_{s b}(p)}{\left|\mathbf{e}_{1} \times \mathbf{e}_{2} \cdot \mathbf{e}_{3}\right|}=\frac{V_{s b}(p)}{2^{3-\frac{1}{2 p}}\left(2 s+2^{-\frac{1}{2 p}}\right)},
$$

where $V_{s b}(p)$ is the volume of superballs given by

$$
V_{s b}(p)=\frac{2}{p^{2}} B\left(\frac{1}{2 p}, \frac{2 p+1}{2 p}\right) B\left(\frac{1}{2 p}, \frac{p+1}{p}\right),
$$

and $B(x, y)=\Gamma(x) \Gamma(y) / \Gamma(x+y)$ and $\Gamma(x)$ is the Euler Gamma function. 
The packing density $\phi_{\max }$ as a function of deformation parameter $p$ is plotted in Fig. 5. The "right" slope of $\phi_{\max }$ at $p=1$ is given by

$$
a_{+}=-\frac{\pi}{12 \sqrt{2}}\left[24+\ln 8+4 \Psi\left(\frac{1}{2}\right)+2 \Psi\left(\frac{3}{2}\right)-6 \Psi\left(\frac{5}{2}\right)\right]=0.3555 \ldots,
$$

where $\Psi(z)=d[\ln \Gamma(z)] / d z$ is the digamma function. The positive value of $a_{+}$indicates that the initial increase of the packing density is linear in $(p-1)$. As mentioned before, the $\mathbb{C}_{0^{-}}$ lattice packings are only optimal for $p \in\left(1, p_{c}^{*}\right)$, beyond $p_{c}^{*}$ the $\mathbb{C}_{1}$-lattice packings become the densest. For $p>1 \phi_{\max }$ increases dramatically until it reaches unity as the particle shapes becomes more like a cube, which is more efficient at filling space than a sphere. These characteristics stand in contrast to those of the densest known ellipsoid packings, achieved by certain crystal arrangements of congruent spheroids with a two-particle basis, whose packing density as a function of aspect ratios has zero initial slope and is bounded from above by a value of $0.7707 \ldots$. 22]. As we will see in Sec. IV, as $p$ decreases from unity, the initial increase of $\phi_{\max }$ is linear in $(1-p)$. Thus, $\phi_{\max }$ is a nonanalytic function of $p$ at $p=1$, which is consistent with our conclusions about superdisk packings. However it is distinctly different from the optimal spheroid packings, for which $\phi_{\max }$ increases smoothly as the aspect ratios of the semi-axes vary from unity and hence has no cusp at the sphere point [22]. The density of congruent ellipsoid packings (not $\phi_{\max }$ ) has a cusp-like behavior at the sphere point only when the packings are randomly jammed [42]. The distinction between the two systems results from different broken rotational symmetries. For spheroids, the continuous rotational symmetry is only partially broken, i.e., spheroids still possess one rotationally symmetric axis; and the three coordinate directions are not equivalent which facilitates dense non-Bravais packings. For superballs, the continuous rotational symmetry of a sphere is completely broken and the three coordinate directions are equivalently fourfold rotationally symmetric directions of the particle. Thus, a superball is less symmetric but more isotropic than an ellipsoid which apparently prefers dense Bravais lattice packings. The broken symmetry of superballs makes their shapes more efficient in tiling space and thus results in a larger and faster increase in the packing density.

At $p=p_{c}^{*}=1.1509 \ldots$, the two lattice packings have the same density $\phi_{c}^{*}=0.7596 \ldots$ and superballs with $p_{c}^{*}$ possess a two-fold degenerate crystalline maximal density state. The $\mathbb{C}_{0}$ and $\mathbb{C}_{1}$ lattices have distinct group symmetries, thus the optimal packing "jumps" from one structure to another leading to another nonanalytic point in $\phi_{\max }$. This behavior is 
similar with that of optimal superdisk packings.

\section{B. The $\mathbb{C}_{1}$-Lattice Packing}

For superballs with $p \geq p_{c}^{*}$ the densest packings are given by the $\mathbb{C}_{1}$ lattices with threefold rotational symmetry consistent with that of the superballs, see Fig. 6(b) and Fig. 10, Note though the $\mathbb{C}_{1}$-lattice packings are only apparently optimal when $p \geq p_{c}^{*}$, they also exist for all $p \geq 1$. In the packing, each superball has 12 contacting neighbors, among which 6 contacting points are the edge centers and the other 6 are along the surface diagonals, as schematically shown in Fig. 9. At $p=1$, this local packing (the central particle and its 12 neighbors) possessing three-fold rotational symmetry is just the building block of the facecentered cubic lattice packing of spheres. As $p$ increases from unity, the continuous rotational symmetry of a sphere is broken while the three-fold rotational symmetry of the local packing could still be maintained since "cubic" superballs still possess three-fold rotational symmetry [see Fig. 4(b)]. Although the edge contacting points will remain at the centers of the edges, the surface contacting points will continuously follow a path along the surface diagonals as $p$ changes from unity to infinity. As $p$ approaches infinity, the surface contacting points will approach the surface centers; when the superballs becomes a perfect cube at $p=\infty$, the contacting points are degenerate, i.e., the entire edges and surfaces of neighboring particles contact each other, respectively.

The three-fold rotational symmetry of the local packing together with the requirement that each particle has 12 contacting neighbors uniquely determine the global packing lattice. And the packing lattice for a specific $p$ value can be obtained by continuously deforming the face-centered cubic lattice. In particular, the lattice vectors of the $\mathbb{C}_{1}$ are given by

$$
\mathbf{e}_{1}=2^{1-\frac{1}{2 p}} \mathbf{i}+2^{1-\frac{1}{2 p}} \mathbf{j}, \quad \mathbf{e}_{2}=2^{1-\frac{1}{2 p}} \mathbf{i}+2^{1-\frac{1}{2 p}} \mathbf{k}, \quad \mathbf{e}_{3}=2\left(s+2^{-\frac{1}{2 p}}\right) \mathbf{i}-2 s \mathbf{j}-2 s \mathbf{k},
$$

where $s$ is the smallest positive root of the following equation:

$$
\left(s+2^{-\frac{1}{2 p}}\right)^{2 p}+2 s^{2 p}-1=0 .
$$

The packing density is readily computed from 


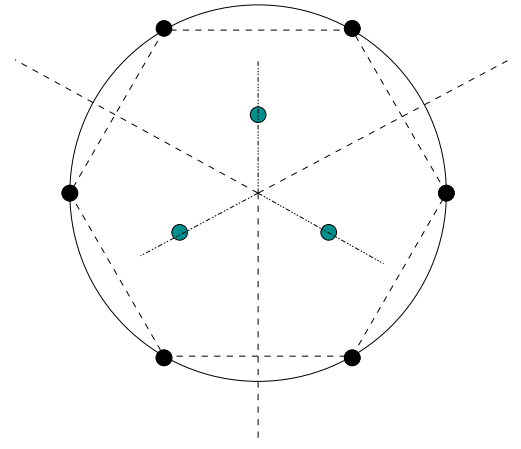

(a)

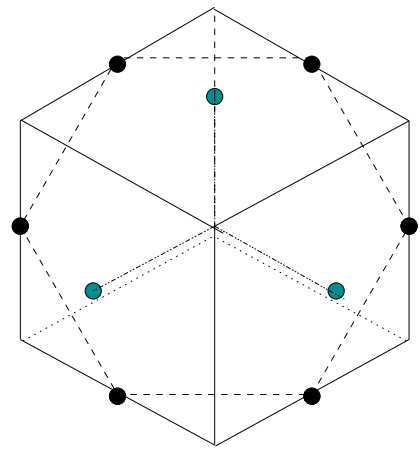

(b)

FIG. 9: (color online). Contacting points of the a sphere (a) and a schematic superball (shown as a cube) (b). The view is along the [111] direction. The surface contacting points (blue) will continuously move along the surface diagonals as the $p$ value changes from unity to infinity. Every contacting point has a symmetric image about the center of the superball and their distributions have three-fold rotational symmetry.

$$
\phi_{\max }=\frac{V_{s b}(p)}{\left|\mathbf{e}_{1} \times \mathbf{e}_{2} \cdot \mathbf{e}_{3}\right|}=\frac{V_{s b}(p)}{2^{3-\frac{1}{p}}\left(3 s+2^{-\frac{1}{2 p}}\right)},
$$

where $V_{s b}(p)$ is the volume of a superball with deformation parameter $p$ as given by Eq. (5) . The packing density $\phi_{\max }$ is shown in Fig. 5,

As shown in Fig. 11(a), a sequence of superballs contacting each other through the centers of edges can be considered as a chain which serves as the basic building block of the packing structure. In contrast to the FCC packing of spheres in which the dense layers constructed by stacking the chains of spheres are parallel to one coordinate plane; for superballs with $p \neq 1$, the chains are shifted relative to each other and the normal direction of the layer containing the centers of superballs does not coincide with the coordinate directions. In this way, a lattice of "pockets" is formed in which the particles in the layer above can be exactly fitted to construct the $\mathbb{C}_{1}$-lattice packing, as illustrated in Fig. 11 (b) and (c). 


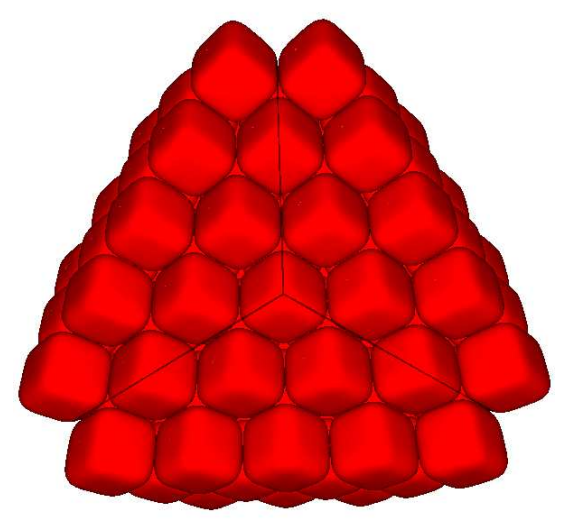

FIG. 10: (color online). The $\mathbb{C}_{1}$ lattice for superballs in the cubic regime viewed from the [111] direction. The thin dark lines show the three orthogonal directions, which clearly exhibits the 3-fold rotational symmetry of the structure.

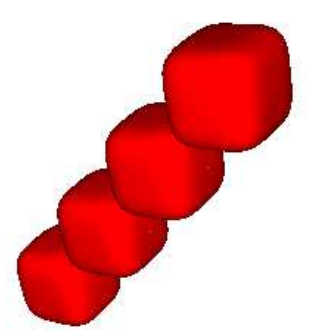

(a)

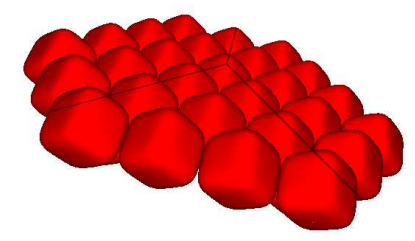

(b)

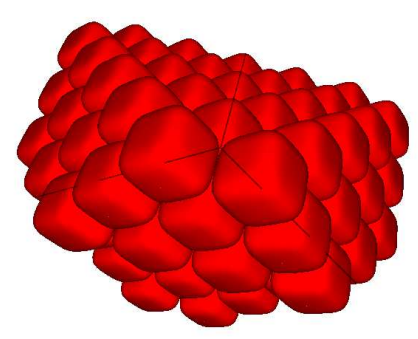

(c)

FIG. 11: (color online). The $\mathbb{C}_{1}$-lattice packing of superballs obtained by stacking superball chains: (a) The chain as depicted in the text. (b) The layer constructed by stacking the chains. (c) The packing obtained by stacking the layers.

\section{OPTIMAL PACKINGS OF SUPERBALLS IN THE OCTAHEDRAL REGIME}

\section{A. The $\mathbb{O}_{0}$-Lattice Packing}

As $p$ decreases from unity, the continuous rotational symmetry of a sphere is broken and a superball has an octahedral-like shape [see Fig. 2(b)]. The $\mathbb{O}_{0}$ lattice [see Fig. [6(c)] also can be considered as a continuous deformation of the face-centered cubic lattice. As before, 


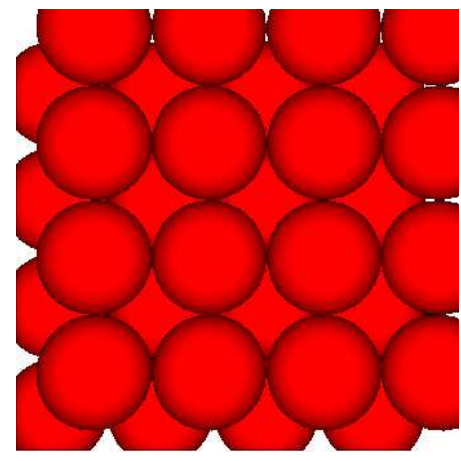

(a)

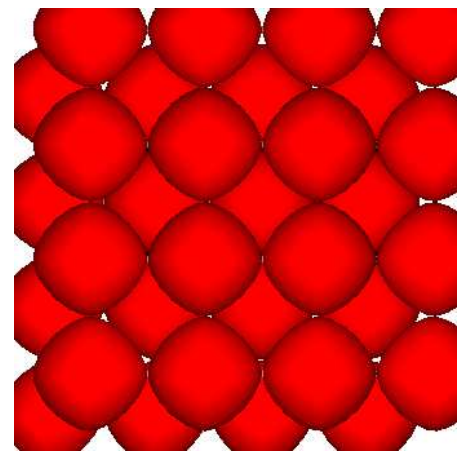

(b)

FIG. 12: (color online). (a) Face-centered cubic packing of spheres, viewed as a laminate of facecentered square layers from the [001] direction. (b) Similar laminate of face-centered square planar layers of superballs in the octahedral regime, viewed from the [001] direction.

we start from the FCC packing, viewed as a laminate of square-lattice planar layers of spheres, as illustrated in Fig. 12(a). We similarly construct layers of superballs by orienting one of the four-fold rotationally symmetric axis along the normal of the layer [see Fig. 4(c)], and aligning the other two of each superball so that the square symmetry of cross-sections of superballs in the layer is consistent with that of the layer, as shown in Fig. 12(b). The layers are stacked so that the superballs in the top layers can exactly sit in the holes formed in the bottom layer. The layer lamination is then continued ad infinitum to generate the packing.

In the $\mathbb{O}_{0}$-lattice packing, each superball has 4 contacting neighbors in its own layer, 4 in the layer above and 4 in the layer below. The lattice vectors for any specific $p$ can be uniquely determined by the symmetry and contacting requirements. i.e.,

$$
\mathbf{e}_{1}=2 \mathbf{i}, \quad \mathbf{e}_{2}=2 \mathbf{j}, \quad \mathbf{e}_{3}=\mathbf{i}+\mathbf{j}+2\left(1-2^{1-2 p}\right)^{\frac{1}{2 p}} \mathbf{k}
$$

As the shape of a superball deviates more from a sphere, the holes in the layer become larger and the distance between successive layers becomes smaller. In the limit that the superballs become octahedra $(p=0.5)$, the planes of two successive layers coincide with each other. However, the distance between every other layer cannot be smaller than 2, which is the nearest distance between centers of two superballs aligned along one of the 
coordinate directions. As $p$ decreases from unity, there exists a point $p_{o}^{*}$ where the particles of every other layer contact each other, forming "cages" for the particles of the layer in between. The value of $p_{o}^{*}$ can be obtained from the relation.

$$
2\left(1-2^{1-2 p}\right)^{\frac{1}{2 p}}-1=0
$$

and we have $p_{o}^{*}=\ln 3 / \ln 4=0.7924 \ldots$ with the corresponding packing density $\phi_{o}^{*}=$ $0.7959 \ldots$. . If $p$ decreases further (i.e., $p<p_{o}^{*}$ ), the "cages" will be too large for the superballs to maintain 12 contacts and the packing density would decrease.

Thus, the $\mathbb{O}_{0}$ lattice only gives the densest known packings of superballs in the vicinity left of the sphere point, i.e., $p_{o}^{*}<p<1$. The packing density is given by

$$
\phi_{\max }=\frac{V_{s b}(p)}{\left|\mathbf{e}_{1} \times \mathbf{e}_{2} \cdot \mathbf{e}_{3}\right|}=\frac{V_{s b}(p)}{8\left(1-2^{1-2 p}\right)^{\frac{1}{2 p}}},
$$

where $V_{s b}(p)$ is the volume of a superball given by Eq. (5) . Packing density $\phi_{\max }$ as a function of $p$ is plotted in Fig. 5 (the branch with $p_{o}^{*}<p<1$ ). The "left" slope of $\phi_{\max }$ at $p=1$ is given by

$$
a_{-}=-\frac{\pi}{12 \sqrt{2}}\left[\ln (64)+8+4 \Psi\left(\frac{1}{2}\right)+2 \Psi\left(\frac{3}{2}\right)-6 \Psi\left(\frac{5}{2}\right)\right]=-0.02941 \ldots
$$

Thus, the initial increase of the packing density as $p$ decreases from unity is linear in $(1-$ $p)$. As pointed out in the previous section, the linear dependence on $|p-1|$ indicates the nonanalyticity of $\phi_{\max }$ at $p=1$, resulting from the broken symmetry of superballs which is distinctly different from other known hard particle systems such as ellipsoids in three dimensions. Note that the magnitude of $a_{-}$is smaller than $a_{+}$, implying that superballs in the cubic regime are more efficient at filling space than superballs in the octahedral regime, as can be also seen in the limiting cases: cubes can tile space while the densest known packing of regular octahedra covers about $94.74 \%$ of the space. The fact that the density of the $\mathbb{O}_{0}$ lattice packing begins to decrease when $p<p_{o}^{*}$ suggests the existence of packings with different symmetry that would be optimal near the octahedron point. 


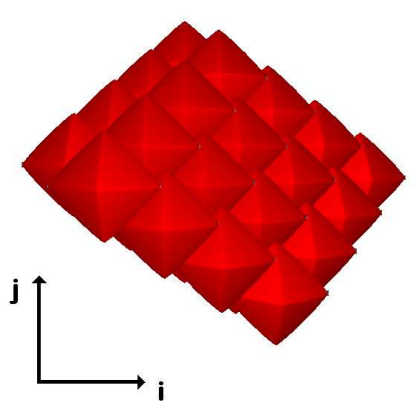

(a)

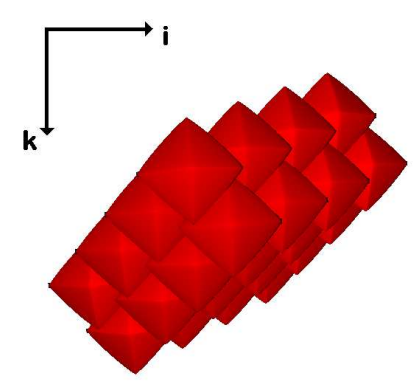

(b)

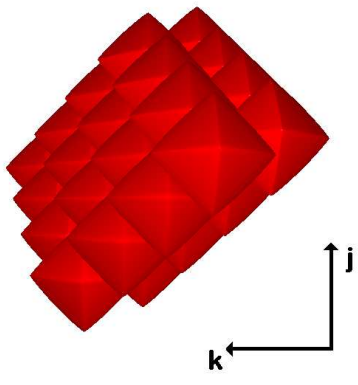

(c)

FIG. 13: (color online). Projections of a $\mathbb{O}_{1}$-lattice sub-packing on three orthogonal coordinate planes: (a) Projection on the (001) plane. (b) Projection on the (010) plane. (c) Projection on the (100) plane.

\section{B. The $\mathbb{O}_{1}$-Lattice Packing}

In particular, the densest known packing of regular octahedra, defined by $\left|x_{1}\right|+\left|x_{2}\right|+$ $\left|x_{3}\right| \leq 1$, is achieved by a Bravais lattice packing with the lattice vectors $[33]$

$$
\mathbf{e}_{1}=\frac{2}{3} \mathbf{i}+\frac{2}{3} \mathbf{j}-\frac{2}{3} \mathbf{k}, \quad \mathbf{e}_{2}=-\frac{1}{3} \mathbf{i}+\frac{4}{3} \mathbf{j}-\frac{1}{3} \mathbf{k}, \quad \mathbf{e}_{3}=\frac{1}{3} \mathbf{i}-\frac{1}{3} \mathbf{j}-\frac{4}{3} \mathbf{k} .
$$

This packing, with density $\phi_{M}=18 / 19=0.9473 \ldots$, was discovered by Minkowski [32]. By perturbing the Minkowski lattice packing using the method depicted in Sec. II.B, we are able to identify a family of highly dense packings of superballs near the octahedron point, i.e., the $\mathbb{O}_{1}$-lattice packing [see Fig. $\left.6(\mathrm{~d})\right]$. As shown in Fig. [13, the projections of the $\mathbb{O}_{1}$-lattice packing on the three orthogonal coordinate planes possess certain translational symmetry in two dimensions. The translational vectors are along $(\mathbf{i}+\mathbf{j})$ direction for $(001)$ plane, along $(\mathbf{i}+\mathbf{k})$ and $(\mathbf{k}-\mathbf{i})$ directions for (010) plane and along $(\mathbf{k}-\mathbf{j})$ direction for (100) plane. The magnitude of the translational vectors are functions of $p$, which can be determined with the additional condition that each particle has 12 contacting neighbors. Thus, we obtain the lattice vectors for the $\mathbb{O}_{1}$, i.e.,

$$
\mathbf{e}_{1}=2 l \mathbf{i}+2 l \mathbf{j}-2 l \mathbf{k}, \quad \mathbf{e}_{2}=-2 q \mathbf{i}+2 s \mathbf{j}-2 q \mathbf{k}, \quad \mathbf{e}_{3}=2 q \mathbf{i}-2 q \mathbf{j}-2 s \mathbf{k},
$$


where $l, s$ and $q$ are given by the following equations

$$
\begin{gathered}
l=3^{-\frac{1}{2 p}}, \quad 2 q^{2 p}+s^{2 p}-1=0, \\
(l+q)^{2 p}+(s-l)^{2 p}+(l-q)^{2 p}-1=0 .
\end{gathered}
$$

At the limit $p=0.5$, Eq. (15) reduces to Eq. (14).

As $p$ increases from 0.5 , the superballs deviate more and more from regular octahedra and it becomes more difficult to maintain 12 contacts for each particle and simultaneously to keep the translational symmetry of the projected packings. Interestingly, as $p$ exceeds $p_{o}^{*}=\ln 3 / \ln 4$, Eq. (16) will have no real roots, indicating that the symmetry and contacting conditions could not be satisfied at the same time beyond $p_{o}^{*}$. Thus, the $\mathbb{O}_{1}$ lattice gives the densest known packings of superballs near the octahedron point, i.e., $0.5<p<p_{o}^{*}$. At $p_{o}^{*}$, the $\mathbb{O}_{0^{-}}$and $\mathbb{O}_{1}$-lattice packings have exactly the same density, i.e., $\phi_{o}^{*}=0.7959 \ldots$ and superballs with $p=p_{o}^{*}$ possess a two-fold degenerate crystalline maximal density state. Note that the $\mathbb{O}_{0}$ and $\mathbb{O}_{1}$ lattices possess distinct symmetries, as $p$ passes $p_{o}^{*}$ the apparently optimal packing "jumps" from one lattice to the other, which results in one more nontrivial nonanalytic point in $\phi_{\max }$. This additional nonanalytical point does not exist for superdisk packings. Nor does similar behavior exist for any other known hard-particle systems.

We emphasize that the $\mathbb{O}_{1}$ - and $\mathbb{O}_{0}$-lattice packings are separately constructed from the FCC packing of spheres which has been proved to be optimal, and from the Minkowski packing - the densest known packing of regular octahedra, respectively. The amazing fact that the two families of packings both terminate at $p_{o}^{*}$ with the same density suggests that these packings are very likely optimal, although we could not completely exclude the possibility that a periodic packing with a complex particle basis might have a higher density over an interval including $p_{o}^{*}$. In other words, the existence of the continuous "path" of superball packings that we found connecting the FCC lattice packing of spheres and the Minkowski lattice packing of regular octahedra given the optimal packings of two limiting particle shapes strongly suggests that the packings along the path are also optimal.

The packing density $\phi_{\max }$ is given by

$$
\phi_{\max }=\frac{V_{s b}(p)}{\left|\mathbf{e}_{1} \times \mathbf{e}_{2} \cdot \mathbf{e}_{3}\right|}=\frac{V_{s b}(p)}{8 l\left(3 q^{2}+s^{2}\right)},
$$

where $V_{s b}(p)$ is the volume of a superball given by Eq. (5). The plot of $\phi_{\max }$ as a function of $p$ is shown in Fig. 5 (the branch with $0.5<p<p_{o}^{*}$ ). The packing structure could also be 


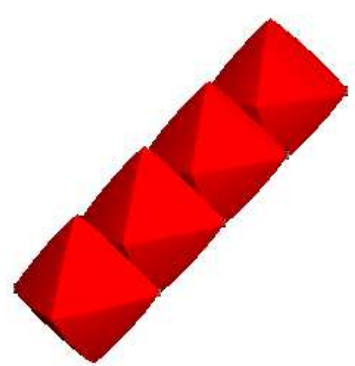

(a)

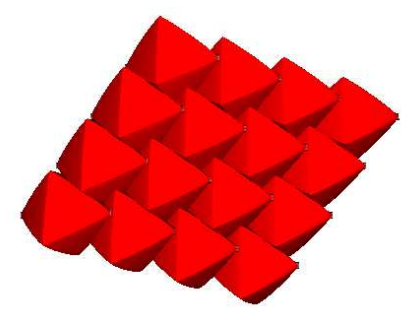

(b)

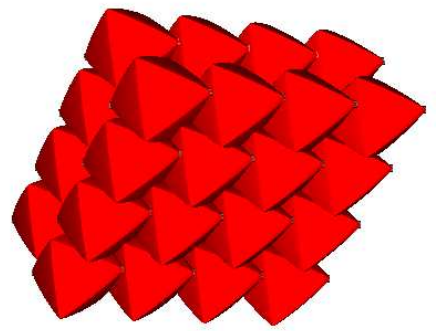

(c)

FIG. 14: (color online). The $\mathbb{O}_{1}$-lattice packing of superballs obtained by stacking superball chains: (a) The superball chain. (b) The layer constructed by stacking the chains. (c) The packing obtained by stacking the layers.

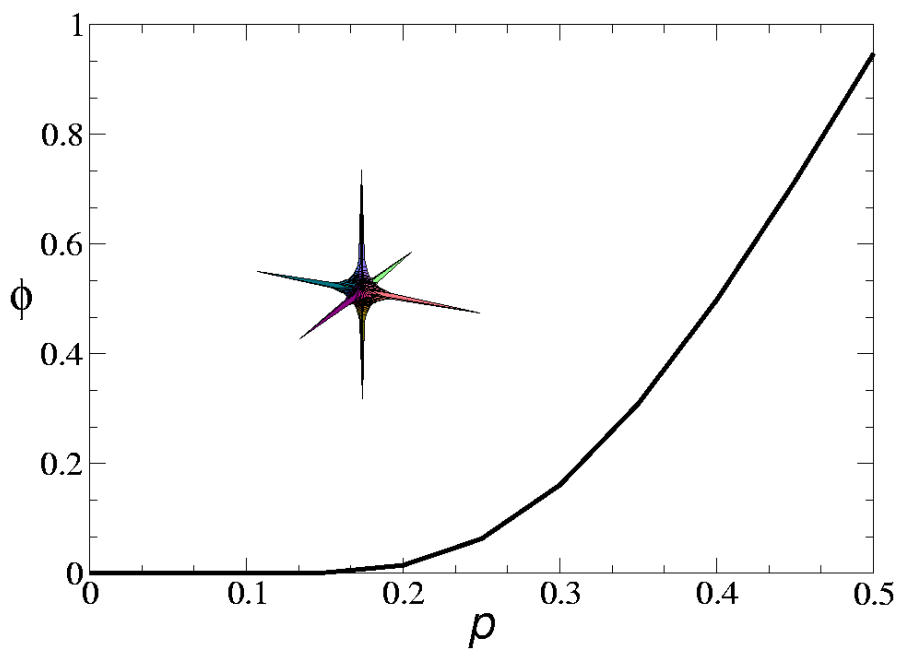

FIG. 15: (color online). Density versus deformation parameter $p$ for the packings of concave superballs. Insert: a concave superball with $p=0.1$, which will becomes a three-dimensional cross at the limit $p \rightarrow 0$.

considered as the stacking of superball chains, as illustrated in Fig. 14.

\section{Generalization to Concave Superballs}

As $p$ decreases from 0.5, the superballs become concave particles, but they still possess octahedral-like shapes [see Fig. 2(a)]. The lack of simulation techniques to generate concave superball packings make it very difficult to find the optimal packings for the entire range 
of concave shapes $(0<p<0.5)$. However, based on our conclusions for convex superball packings, we conjecture that near the octahedron point, the optimal packings possess similar translational symmetry in two dimensions to that of the $\mathbb{O}_{1}$-lattice packing. By inserting into each octahedron in the Minkowski packing a concave superball, we are able to obtain a family of packings that are dense near $p=0.5$ but not optimal, the density of which is shown in Fig. 15. The packings could be densified a little by carefully adjusting the particles to minimize the exclusion volume effects. However, the adjustments are difficult to quantify.

It is interesting to point out that at the limit $p \rightarrow 0$, one can construct zero-length "chains" of a infinite number of "crosses" along the [111] (or any equivalent) direction. In other words, the centers of "crosses" packed along the chain are infinitely close to each other. This aligning effect, which appears in three dimensions but not in two dimensions, suggests that near the "cross" point, the similarly constructed chains of superballs might be a reasonable building block to produce dense packings.

\section{CONCLUSIONS}

In this paper, we have constructed the densest known packings of convex superballs based on symmetry and contacting requirements. For superballs in the cubic regime $(p>1)$, the candidate optimal packings are achieved by the $\mathbb{C}_{0^{-}}$and $\mathbb{C}_{1}$-lattice packings possessing twofold and three-fold rotational symmetry, respectively, which can both be considered as a continuous deformation of the FCC lattice. For convex superballs in the octahedral regime $(0.5<p<1)$, the $\mathbb{O}_{1}$ - and $\mathbb{O}_{0}$-lattice, obtainable from continuous deformation of the FCC lattice keeping its four-fold rotational symmetry, and from the Minkowski lattice for regular octahedra [32] keeping the translational symmetry of the projected lattice on the coordinate planes, are apparently optimal in the vicinity of the sphere- and the octahedron-point, respectively. The packings jump between distinct lattices as $p$ passes $p_{c}^{*}$ and $p_{o}^{*}$, leading to two nontrivial nonanalytic points in $\phi_{\max }$.

The existence of the continuous "path" of superball packings that we found connecting the FCC lattice packing of spheres and the Minkowski lattice packing of regular octahedra provides strong evidence that our candidate packings are very likely optimal. For concave superballs, we constructed a family of dense packings based on the Minkowski lattice and discussed an interesting aligning effect. Moreover, we have shown that as $p$ changes from unity, 
there is a significant increase of the maximal packing density $\phi_{\max }$, which is initially linear in $|p-1|$ resulting in the nonanalytic behavior of $\phi_{\max }$ at the sphere point. Interestingly, this three-dimensional behavior is consistent with the fact that there is an exponential improvement on the lower bound on $\phi_{\max }$ of superballs relative to that for spheres in arbitrarily high dimensions as found by Elkies, Odlyzko and Rush [27]. The packing characteristics of superball packings are much richer than those of superdisks, and the nontrivial influences of the broken symmetry are distinctly different from the other known aspherical packings, such as ellipsoid packings. Although the anisotropic shape of the particle allows for the possibility of local arrangements with larger coordination numbers, in the candidate optimal packings each superball maintains twelve contacting neighbors for all values of $p$, in contrast to the densest known ellipsoid packings in which each particle has fourteen contacting neighbors.

The optimal Bravais lattice packings of convex superballs for a particular value of $p$ are also the corresponding dense crystal phase states of superballs in equilibrium. Therefore, our findings provide a starting point to quantify the entire equilibrium phase behavior of superball systems, which should deepen our understanding of the statistical thermodynamics of non-spherical particle systems [44, 45, 46]. Ideally, one would expect a first-order disorder-order phase transition of superball systems for every value of $p$. However, the kinetic difficulty of such a transition increases with the geometrical frustrations introduced by the particles. In practice, the disordered metastable states could frequently occur, as illustrated in Fig. 3(b). Some preliminary investigations that we have carried out show that disordered superball packings are hypoconstrained, i.e., the average contact number is smaller than twice of the number of degrees of freedom of the particles [47]. A rotational transition is also possible, i.e., the rotational degrees of freedom of the particles are suddenly frozen when the packing density exceeds a certain threshold value. A unique feature of superball systems is that symmetry transitions occur at $p_{c}^{*}$ and $p_{o}^{*}$. Thus for particles with a deformation parameter close to $p_{c}^{*}$ or $p_{o}^{*}$, multi-morphological maximal density states might be found.

Moreover, the optimal packings of convex superballs are significantly denser than optimal sphere packings when $p$ deviates considerably from unity; even the disordered packings could have a densities close to $\phi_{\max }$ of spheres. This suggests that one could use particles with shapes similar to that of superballs in situations where dense packings are favored, such as in certain powder sintering processes. We also note that superballs can be mass produced 


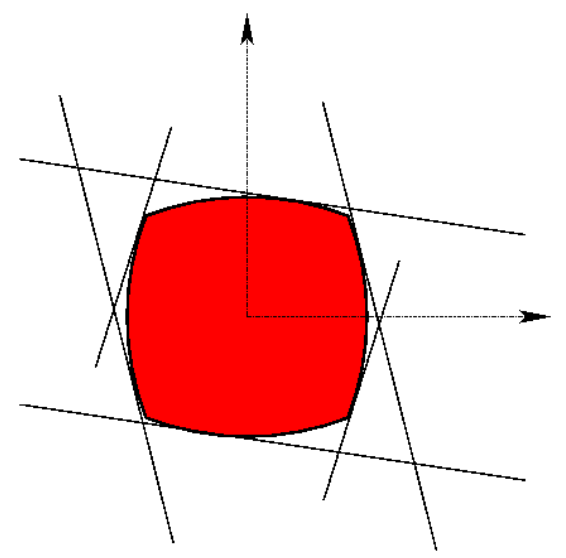

FIG. 16: (color online). A superdisk circumscribed by a hexagon formed from three pairs of parallel lines.

using modern lithography techniques, which would enable one to prepare and study superball packings experimentally.

In the future, we will generalize the current work to the study of disordered packings of superdisks and superballs and further explore the effect of broken rotational symmetry. It will be also interesting to understand the "jamming" characteristics [43, 47] of both random and crystalline packings of these particles.

\section{APPENDIX A: OPTIMALITY OF THE SUPERDISK PACKINGS FOUND IN REF. [30]}

In this section, we briefly outline the procedure used to verify that for every specific value of $p>0.5$ the constructed packings of superdisks in two dimensions in Ref. [30] are indeed optimal, by virtue of Fejes Toth's theorem [12, 13] described in Sec. I. In particular, Fejes Toth's theorem states that the densest packing of any centrally symmetric convex body in two dimensions is always given by the smallest hexagon that circumscribes the body. Since the body is centrally symmetric, the hexagon is as well and therefore it tiles $\mathbb{R}^{2}$. Fejes Toth's theorem also implies that the optimal packing for any two-dimensional centrally symmetric body is a Bravais lattice packing. Thus, the problem of finding the optimal packing reduces to finding the circumscribing hexagon of a superdisk with minimum area.

Let a superdisk be circumscribed by three pairs of parallel lines forming a hexagon, as shown in Fig. 16. Denote the common tangent points by $M_{i}$ with the coordinates $\left(m_{1}^{(i)}, m_{2}^{(i)}\right)$ 
$(i=1, \ldots, 6)$. Since the particle is centrally symmetric, $M_{i}$ and $M_{i+3}(i=1,2,3)$ are also symmetric about the center, i.e., $m_{j}^{(i)}=-m_{j}^{(i+3)}(j=1,2)$.

From the common tangent conditions, one can write down the equations of the parallel lines in terms of the coordinates $m_{j}^{(i)}(i=1, \ldots, 6, j=1,2)$, from which the vertices of the hexagon as intersections of different pairs of lines can be obtained. Thus, the area of

the hexagon is readily expressed as a function of $m_{j}^{(i)}$, i.e., $S\left(m_{1}^{(1)}, \ldots, m_{2}^{(6)}\right)$. By solving the following optimization problem

$$
\begin{gathered}
\text { Minimize: } \quad S\left(m_{1}^{(1)}, \ldots, m_{2}^{(6)}\right), \\
\text { Subject to: } \quad\left|m_{1}^{(i)}\right|^{2 p}+\left|m_{2}^{(i)}\right|^{2 p}=1, \quad(i=1, \ldots, 6) ;
\end{gathered}
$$

one can find the smallest hexagon circumscribing the particle and thus verify for every specific $p>0.5$, the packings given in Ref. [30] are indeed optimal.

\section{ACKNOWLEDGMENTS}

The authors would like to thank Aleksandar Donev, Martin Henk and Greg Kuperberg for valuable discussions. S. T. thanks the Institute for Advanced Study for its hospitality during his stay there. This work was supported by the Division of Mathematical Sciences at the National Science Foundation under Award Number DMS-0804431 and by the MRSEC Program of the National Science Foundation under Award Number DMR-0820341.

[1] R. Zallen, The Physics of Amorphous Solids (Wiley, New York, 1983).

[2] P. M. Chaikin and T. C. Lubensky, Principles of Condensed Matter Physics (Cambridge University Press, New York, 2000).

[3] I. C. Kim and S. Torquato, J. Appl. Phys. 69, 2280 (1991); S. Torquato, J. Mech. Phys. Solids 46, 1411 (1998); M. C. Rechtsman and S. Torquato, J. Appl. Phys. 103, 084901 (2008).

[4] S. Torquato, Random Heterogeneous Materials: Microstructure and Macroscopic Properties (Springer-Verlag, New York, 2002).

[5] T. Zohdi, Int. J. Numer. Meth. Eng. 76, 1250 (2008).

[6] S. F. Edwards, Granular Matter (Ed. A. Mehta, Springer-Verlag, New York, 1994). 
[7] W. D. Callister, Materials Science and Engineering: An Introduction, 7th Edition (Wiley, New York, 2006).

[8] J. Liang and K. A. Dill, Biophys J. 81, 751 (2001).

[9] J. W. S. Cassels, An Introduction to the Geometry of Numbers (Springer-Verlag, Berlin, 1959).

[10] J. H. Conway and N. J. A. Sloane, Sphere Packings, Lattices and Groups (Springer, Berlin Heidelberg New York, 1987).

[11] C. E. Shannon, Bell System Tech. J. 27, 379 (1948); ibid., 623 (1948).

[12] L. Fejes Tóth, Regular Figures (Macmillan, New York, 1964).

[13] J. Pach and P. K. Agarwal, Combinatorial Geometry (Wiley-Interscience, New York, 1995).

[14] T. C. Hales, Ann. Math. 162, 1065 (2005).

[15] H. Cohn and N. Elkies, Ann. Math. 157, 689 (2003).

[16] G. Parisi and F. Zamponi, J. Stat. Mech.: Theory Exp. P03017 (2006).

[17] S. Torquato and F. H. Stillinger, Exp. Math. 15, 307 (2006).

[18] G. Parisi, J. Stat. Phys. 132, 207 (2008).

[19] A. Scardicchio, F. H. Stillinger, and S. Torquato, J. Math. Phys. 49, 043301 (2008).

[20] Supramolecular chemistry deals with the chemistry and collective behavior of molecular building blocks that are organized on large length scales (relative to molecular sizes) with long-range order.

[21] A. I. Kitaigorodskii, Molecular Crystal and Molecules, (Academic Press, New York, 1973).

[22] A. Donev, F. H. Stillinger, P. M. Chaikin, and S. Torquato, Phys. Rev. Lett. 92, 255506 (2004).

[23] J. H. Conway and S. Torquato, Proc. Nat. Acad. Sci. 103, 10612 (2006).

[24] P. Chaikin, S. Wang, and A. Jaoshvili, Abstract submitted to APS March Meeting (2007).

[25] E. R. Chen, Discrete Comput. Geom. 40, 214 (2008).

[26] It is highly likely that the largest density of tetrahedral packings reported in Ref. [25] can be improved.

[27] N. D. Elkies, A. M. Odlyzko, and J. A. Rush, Invent. math. 105, 613 (1991).

[28] There is a fundamental distinction between the shapes of superball surfaces in the octahedrallike regime $(0 \leq p<1)$ and the cubic-like regime $(1<p \leq+\infty)$. The former display a set of linear loci along which a principal curvature diverges, representing a generalization of the edge set for the true octahedron at $p=1 / 2$. No such loci of diverging curvature exist for the 
cubic-like regime, except in the limit $p \rightarrow+\infty$.

[29] Two polyhedra are dual to each other if the vertices of one correspond to the faces of the other.

[30] Y. Jiao, S. H. Stillinger, and S. Torquato, Phys. Rev. Lett. 100, 245504 (2008).

[31] J. A. Rush, Invent. Math. 98, 499 (1989).

[32] H. Minkowski, Nachr. K. Ges. Wiss. Göttingen, Math.-Phys. KL, 311 (1904).

[33] U. Betke and M. Henk, Comput. Geom. 16, 157 (2000). This paper reports the densest Bravais lattice packings of a number of different three-dimensional polyhedra.

[34] The regular octahedron is centrally symmetric. In the densest octahedral lattice packing found by Minkowski [32], the octahedra have the maximal number of face-to-face contacting neighbors, which significantly reduces the free volume. This observation together with the results of our optimization simulations described in the text strongly suggests that the Minkowski lattice packing of regular octahedra is optimal among all packings of octahedra, which we conjecture to be the case.

[35] A. Donev, S. Torquato, and F. H. Stillinger, J. Comput. Phys. 202, 737 (2005).

[36] B. D. Lubachevsky and F. H. Stillinger, J. Stat. Phys. 60, 561 (1990).

[37] S. Torquato, T. M. Truskett, and P. G. Debenedetti, Phys. Rev. Lett. 84, 2064 (2000).

[38] A. R. Kansal, S. Torquato, and F. H. Stillinger, Phys. Rev. E 86, 041109 (2002).

[39] In a saturated packing, there is no space available to add another particle to the packing.

[40] A. Donev, Jammed Packings of Hard Particles, Ph.D. Thesis, Princeton University, 2006.

[41] Y. Jiao and S. Torquato, in preparation.

[42] A. Donev, I. Cisse, D. Sachs, E. A. Variano, F. H. Stillinger, R. Connelly, S. Torquato, and P. M. Chaikin, Science 303, 990 (2004).

[43] S. Torquato and F. H. Stillinger, J. Phys. Chem. B 105, 11849 (2001).

[44] K. W. Wojciechowski and D. Frenkel, Comput. Methods Sci. Technol. 10, 235 (2004).

[45] A. Donev , J. Burton, F. H. Stillinger, and S. Torquato, Phys. Rev. B 73, 054109 (2006).

[46] G. Yatsenko and K. S. Schweizer, Langmuir 24, 7474 (2008).

[47] A. Donev, R. Connelly, F. H. Stillinger, and S. Torquato, Phys. Rev. E 75, 051304 (2007). 

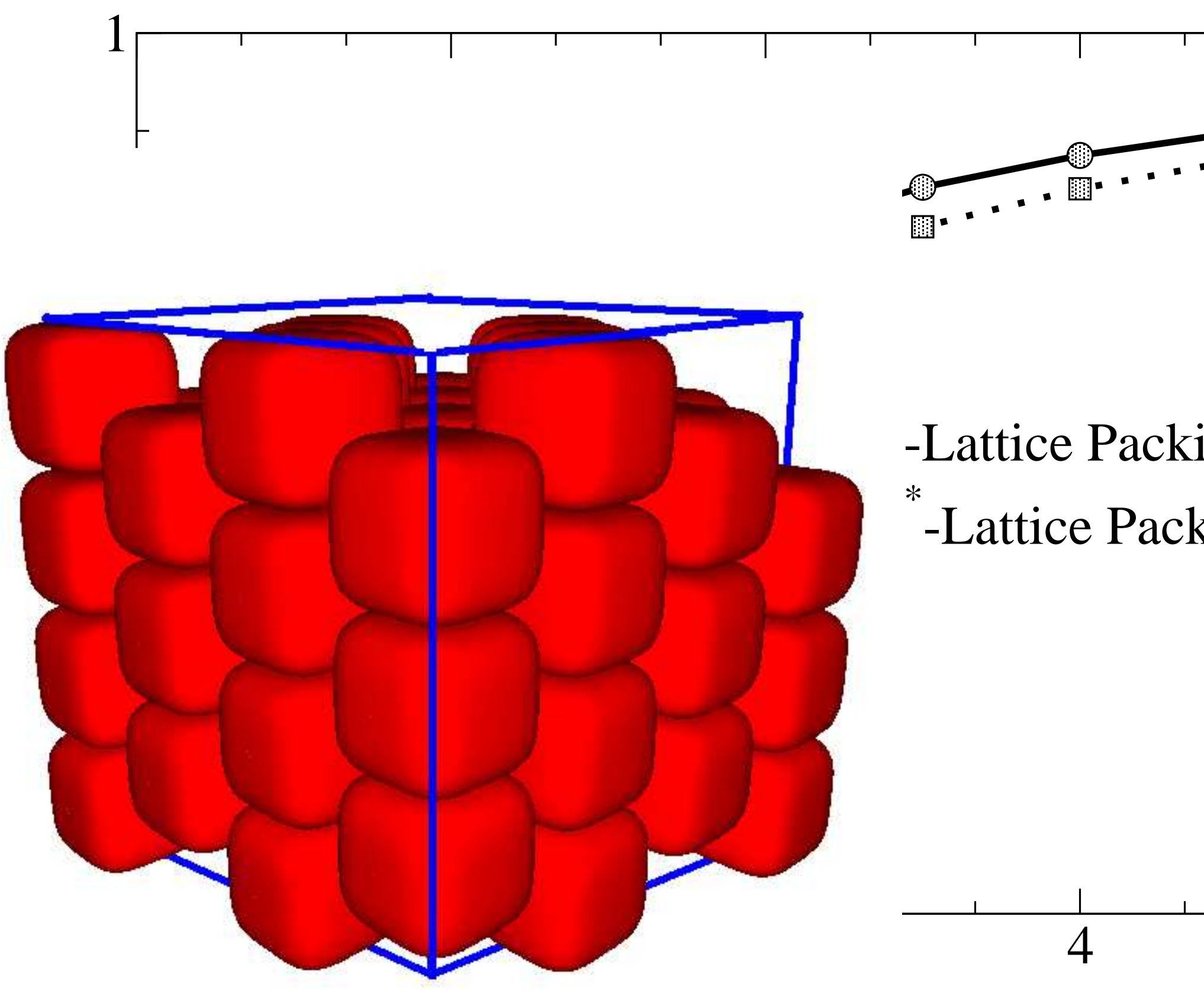

-Lattice Packi *

-Lattice Pack 